\title{
ECG Evaluator Blinding Parameters
}

National Cancer Institute

\section{Source}

National Cancer Institute. ECG Evaluator Blinding Parameters. NCI Thesaurus. Code C119560.

A description of the parameters to which the ECG assessors were blinded to within the study. 